\title{
Evaluation of the expression of fatty acid synthase and $O$-GlcNAc transferase in patients with liver cancer by exploration of transcriptome databases and experimental approaches
}

\author{
SADIA RAAB ${ }^{1}$, NINON VERY ${ }^{1}$, BELINDA DUCHÊNE ${ }^{2}$, PIERRE RYBARCZYK ${ }^{3}$, \\ NICOLAS JONCKHEERE ${ }^{2}$, IKRAM EL YAZIDI-BELKOURA ${ }^{1}$ and TONY LEFEBVRE ${ }^{1}$
}

${ }^{1}$ Université de Lille, CNRS, UMR 8576-UGSF-Unité de Glycobiologie Structurale et Fonctionnelle; ${ }^{2}$ Université de Lille, CNRS, Inserm, CHU Lille, UMR 9020-U1277, CANTHER-Cancer Heterogeneity, Plasticity and Resistance to Therapies, F-59000 Lille; ${ }^{3}$ Service d'Anatomie et Cytologie Pathologique, CHU Amiens-Picardie, F-80000 Amiens, France

Received July 22, 2021; Accepted December 8, 2021

DOI: 10.3892/ol.2022.13225

\begin{abstract}
Tumor occurrence and development are closely related to metabolism abnormalities. One of the metabolic networks that is dysregulated during carcinogenesis is the fatty acid synthesis pathway, which is mainly controlled by fatty acid synthase (FASN). We previously demonstrated in proliferating HepG2 liver cancer cells that FASN expression depends on the catalytic activity of $O$-GlcNAc transferase (OGT) and the activation of the mechanistic/mammalian target of rapamycin (mTOR) pathway. The aim of the present study was to go further in these investigations by analyzing datasets and tissues of patients with liver cancer. To that purpose, transcriptome databases were explored, and reverse transcription-quantitative PCR, western blotting and immunohistochemistry were used. Database analyses revealed that FASN and OGT gene expression was higher in certain cancer tissues, including liver hepatocellular carcinoma, compared with that in non-cancerous tissues. At the protein level, FASN expression was higher in the liver cancer-derived cell lines HepG2 and Hep3B compared with the immortalized human hepatocytes IHH cell line. However, neither the expression of OGT nor of its product $O$-GlcNAcylation showed any significant difference among the three hepatic cell lines. Subsequently, the expression of FASN and OGT at the protein and mRNA
\end{abstract}

Correspondence to: Professor Tony Lefebvre, Université de Lille, CNRS, UMR 8576-UGSF-Unité de Glycobiologie Structurale et Fonctionnelle, F-59000 Lille, France

E-mail: tony.lefebvre@univ-lille.fr

Abbreviations: FASN, fatty acid synthase; GlcNAc, $\mathrm{N}$-acetylglucosamine; HCC, hepatocellular carcinoma; IHH, immortalized human hepatocytes; mTOR, mechanistic/mammalian target of rapamycin; OGA, $O$-GlcNAcase; OGT, $O$-GlcNAc transferase; SREBP, sterol responsive element binding protein

Key words: fatty acid synthase, $O$-GlcNAc transferase, $O$-GlcNAcylation, liver cancer, mTOR pathway levels was evaluated in human liver cancer and non-tumoral tissues from the same patients with different liver lesions. The results from western blotting demonstrated a significant increase in OGT ands $O$-GlcNAcylation expression in liver cancer tissues independently of the type of lesion characterizing the non-tumoral counterpart. As previously reported for HepG2 proliferating cells, the protein level of FASN was positively correlated with the activation of mTOR and, although a rather upward trend, a high variability in its expression was monitored between patients. However, the results from immunohistochemistry showed no particular modification for OGT and $O$-GlcNAcylation expression and a significant increase in FASN expression in cancer tissues compared with that in adjacent non-tumoral tissues. Non-significant changes were observed for FASN and OGT mRNA levels between tumoral and non-tumoral samples, with a high variability between patients. Taken together, these results demonstrated that FASN expression was higher in hepatic cancer tissues in comparison with non-tumoral tissues. Furthermore, OGT expression and activity were shown to vary greatly between cell or cancer type, making any generalization difficult.

\section{Introduction}

Fatty acid synthase (FASN) synthesizes fatty acids from malonyl-CoA and acetyl-CoA substrates, using nicotinamide adenine dinucleotide phosphate $\left(\mathrm{NADPH}, \mathrm{H}^{+} / \mathrm{NADP}^{+}\right)$as a cofactor, mainly leading to the synthesis of 16-carbon palmitate (1). FASN is also involved in other functions such as energy storage, protein adhesion to membrane, cell signaling, intracellular trafficking, cell migration and cell proliferation (2). In non-cancerous human tissues or cells, FASN, which is under the transcriptional control of sterol responsive element binding protein (SREBP), is downregulated due to a sufficient level of fatty acids in the diet. Accelerated fatty acid synthesis due to increased FASN level has been observed in many types of cancer, including breast, colon or prostate cancer, and is positively correlated with a poor prognosis (3).

$O$-GlcNAcylation is a dynamic post-translational modification consisting in the addition of a single 
$\mathrm{N}$-acetylglucosamine (GlcNAc) monosaccharide to serine/threonine residues of target proteins via $O$-GlcNAc transferase (OGT). Removal of the GlcNAc residue is catalyzed by the $O$-GlcNAcase (OGA) (4). As a nutrient sensor, $O$-GlcNAcylation can relay the effects of excessive nutritional intake, which is an important risk factor of cancer. It has been reported that $O$-GlcNAcylation and OGT levels are increased in various types of cancers such as colon and breast cancer (5). We previously demonstrated in two independent studies the following: i) FASN is $O$-GlcNAcylated in a nutrition-dependent manner (hepatic lipogenesis) and $O$-GlcNAcylation promotes its activity by preventing its proteasomal degradation (6); and ii) FASN expression is dependent of the catalytic activity of OGT and activation of mTOR in proliferating liver cancer cells (7).

mTOR pathway is another signaling pathway that senses nutrient availability and growth factors or hormones to enable cell growth (8). Our previous study and another study reported that $O$-GlcNAcylation and mTOR pathway are closely linked in breast and colon cancer cells, and a reciprocal control between the two has been demonstrated $(9,10)$. Furthermore, it has been reported that the mTOR pathway is associated with tumorigenesis $(11,12)$. However, such investigation in human liver tissues has not been performed.

In a previous study, we focused on the expression of FASN in the HepG2 cell line (7); however, further investigation is needed in patients with liver cancer. By combining the evaluation of transcriptome databases and experimental approach using western blotting, immunohistochemistry (IHC) and reverse transcription quantitative (RT-q)PCR, the present study investigated the expression of FASN and OGT and the activation of mTOR in liver-derived cell lines and tissues from patients with liver cancer. The objective of the present study was to extend the research of our previous study on cell lines (7) and to tentatively fill a gap in the literature concerning the concomitant expression level of FASN and OGT, and mTOR pathway activation, in hepatic cancer.

\section{Materials and methods}

Expression data retrieval and analysis. The OGT and FASN gene expression in tissues was graphed independently of sex and along a logarithmic y-axis $[\log 10(\mathrm{TPM}+1)]$ using Genotype-Tissue Expression (GTEx; https://gtexportal. org/home/) database. The web server GEPIA 2 (cancer-pku. cn; gepia2.cancer-pku.cn) was used to analyze the Cancer Genome Atlas (TCGA) database. GTEx and TCGA together allowed the examination of 60,498 genes and 198,619 isoforms (dataset sources). Expression analyses generated by GEPIA2 were represented as box plots with a cutoff P-value of 0.01 . Log scale was chosen for data representation.

Human tumor tissues. A series of 10 liver tumor and tumor-adjacent tissues from 6 men and 4 women were obtained from the Tumor Bank of Lille-Regional Reference Center in Cancer (Centre Hospitalier Régional Universitaire de Lille, Lille, France; agreement no. \#CSTMT276 obtained on December 2, 2020). Samples were immediately frozen in liquid nitrogen and stored at $-80^{\circ} \mathrm{C}$. Patient data are presented in Table I.
Tissue disruption. Liver tissues were lysed in $600 \mu 1$ of lysis buffer [10 mM Tris- $\mathrm{HCl}, 150 \mathrm{mM} \mathrm{NaCl}, 0.1 \%$ (m/v) sodium dodecyl sulfate (SDS), $1 \%(\mathrm{v} / \mathrm{v})$ Triton-X100 and $0.5 \%(\mathrm{~m} / \mathrm{v})$ sodium deoxycholate ( $\mathrm{NaDOC}) ; \mathrm{pH} 7.5$ ] containing protease inhibitors (protease cocktail inhibitors; Sigma-Aldrich; Merck KGaA), $50 \mathrm{mM}$ sodium fluoride (Sigma-Aldrich; MerckKGaA) and $1 \mathrm{mM}$ sodiumorthovanadate(Sigma-Aldrich; Merck $\mathrm{KGaA}$ ) at $4^{\circ} \mathrm{C}$ for protein extraction or $500 \mu \mathrm{l}$ of RA1 buffer (Machery-Nagel $\mathrm{GmbH}$ ) containing chaotropic salt [30-60\% (m/v) guanidinium thiocyanate] at room temperature for mRNA extraction using a MP Biomedicals Instrument FastPrep and Lysing Matrix tubes (MP Biomedicals). Three cycles of $40 \mathrm{sec}$ at $4 \mathrm{msec}^{-1}, 40 \mathrm{sec}$ at $4 \mathrm{msec}^{-1}$ and $20 \mathrm{sec}$ at $4 \mathrm{msec}^{-1}$ were needed. The soluble fractions were obtained following two centrifugations at $13,000 \mathrm{x}$ g for $10 \mathrm{~min}$ at $4^{\circ} \mathrm{C}$ for proteins and at room temperature for mRNA.

Cell culture. All cell lines were obtained from the American Type Culture Collection apart from the immortalized human hepatocytes IHH cell line that was provided by the European Genomic Institute for Diabetes (Lille). The human liver cancer HepG2 cell line was cultured in Dulbecco's Modified Eagle's Medium (DMEM; Lonza Group Ltd.) supplemented with $25 \mathrm{mM}$ glucose. The human hepatocarcinoma Hep3B cell line was cultured in Minimal Essential Medium (MEM; Biowest SAS) supplemented with $5 \mathrm{mM}$ glucose. The immortalized human hepatocyte IHH cell line was cultured in William's E Medium (Lonza Group Ltd.) supplemented with $10 \mathrm{mM}$ glucose. All cells were maintained in medium supplemented with $10 \%$ (v/v) fetal calf serum (Dominique Dutscher SAS) and $2 \mathrm{mM}$ L-glutamine and incubated at $37^{\circ} \mathrm{C}$ in a $5 \%(\mathrm{v} / \mathrm{v})$ $\mathrm{CO}_{2}$-enriched humidified atmosphere. To maintain optimal growth conditions, cells were divided before confluence was reached and fresh medium was added. The day before cell were used, the cells were divided to retain their ability to proliferate.

Western blotting. Cells were first washed twice with ice-cold phosphate-buffered saline (Sigma-Aldrich; Merck KGaA) and then incubated for $20 \mathrm{~min}$ with lysis buffer (composition as aforementioned). The cell lysates were then centrifuged at $20,000 \mathrm{x} \mathrm{g}$ for $15 \mathrm{~min}$ at $4^{\circ} \mathrm{C}$. The supernatants were collected and protein concentration from cultured cells and human liver lysates was evaluated using the micro-BCA assay kit (Thermo Fisher Scientific, Inc.) according to the manufacturer's instructions. Proteins (30 $\mu \mathrm{g}$ per lane) were separated by 6 or $8 \%$ SDS-PAGE in electrophoresis buffer [25 mM Tris-HCl, $192 \mathrm{mM}$ glycine, 0.1\% (m/v) SDS, pH 8.8] and transferred onto nitrocellulose membranes (Hybond ${ }^{\mathrm{TM}}-\mathrm{C}$ EXTRA; GE Healthcare) in transfer buffer [25 mM Tris- $\mathrm{HCl}$, 192 mM glycine, 20\% (v/v) methanol, $\mathrm{pH} 8,8]$. Membranes were stained with Ponceau red $[5 \%(\mathrm{v} / \mathrm{v})$ acetic acid and $0,1 \%$ (w/v) Ponceau red] to confirm equal loading. Membranes were destained with Tris-Buffered Saline (TBS) containing Tween-20 [20 mM Tris-HCl, $150 \mathrm{mM} \mathrm{NaCl,} \mathrm{0,05 \%} \mathrm{(v/v)}$ Tween-20; Sigma-Aldrich; Merck KGaA; pH 7,5; TBS-T]. Membranes were subsequently blocked in $5 \%(\mathrm{w} / \mathrm{v})$ nonfat dry milk or 3\% (w/v) bovine serum albumin (Sigma-Aldrich; Merck KGaA) in TBS-T for $45 \mathrm{~min}$ and incubated overnight at $4^{\circ} \mathrm{C}$ with primary antibodies against $O$-GlcNAc [mouse monoclonal (RL2); cat. no. MA1-072; Thermo Fisher 


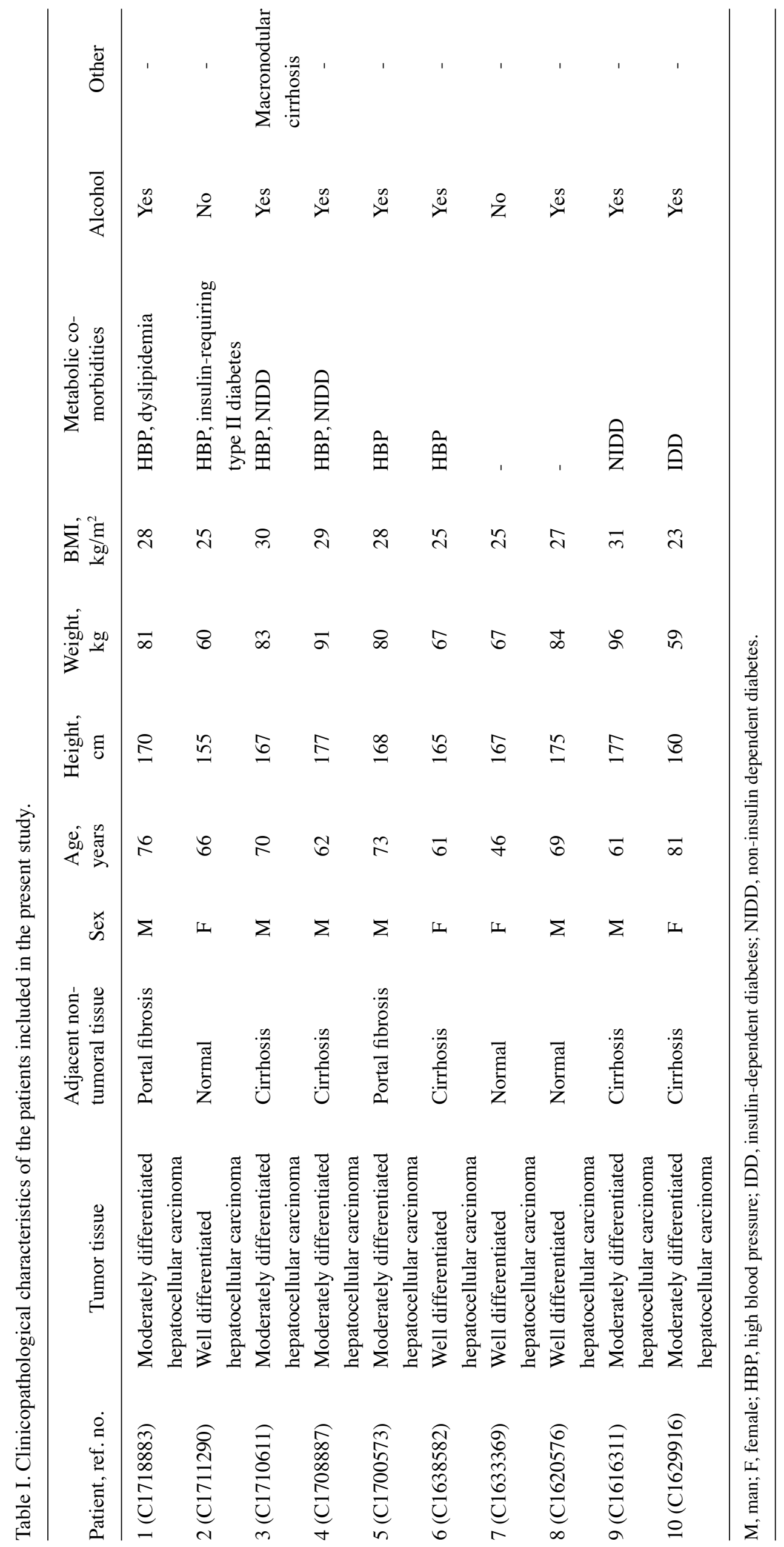


Table II. Sequences of the primers used for reverse transcription quantitative PCR.

Genes

Forward sequence, 5'-3'

Reverse sequence, 5'-3'

Hybridization

\begin{tabular}{llll}
\hline$O G T$ & TGGCTTCAGGAAGGCTATTG & CAAGTCTTTTGGATGTTCATATGG & 56 \\
$F A S N$ & TTCTTCGGAGTCCACCCCA & TCCTCGGAGTGAATCTGGGT & 60 \\
$S R E B P$ & GGAGCCATGGATTGCACTTT & TCAAATAGGCCAGGGAAGTCA & 56 \\
$R P L P O$ & GATGACCAGCCCAAAGGAGA & GTGATGTGCAGCTGATCAAGACT & 60 \\
\hline
\end{tabular}

FASN, fatty acid synthase; OGT, $O$-GlcNAc transferase; SREBP, sterol responsive element binding protein; RLP0, ribosomal protein lateral stalk subunit P0.

Scientific, Inc. 1:2,000], OGT [rabbit polyclonal (TI-14); cat. no. O6014; Sigma-Aldrich; Merck KGaA; 1:2,000], FASN (rabbit polyclonal; cat. no. ab99359, Abcam, 1:1,000), mTOR [rabbit polyclonal (7C10); cat. no. \#2983; Cell Signaling Technology, Inc.; 1:1,000], phosphorylated (p)-mTOR [rabbit polyclonal (D9C2); cat. no. \#5536; Cell Signaling Technology, Inc; 1:1,000] and GAPDH (mouse monoclonal; cat. no. 71548; Covalab; 1:4,000). After three washes with TBS-T, membranes were incubated with the appropriate horseradish peroxidase-conjugated secondary antibody (polyclonal donkey anti-rabbit IgG/HRP conjugated and polyclonal sheep anti-mouse IgG/HRP conjugated; GE Healthcare; 1:10,000) for $1 \mathrm{~h}$ at room temperature. After three washes with TBS-T, bands were detected using enhanced chemiluminescence substrate (West Pico Plus; Thermo Fisher Scientific, Inc.). The images were acquired using a CCD camera (Fusion Solo; Vilbert Lourmat). For additional probing, membranes were stripped with the Antibody Stripping Buffer (Gene Bio-Application L.T.D.) for $15 \mathrm{~min}$ at room temperature, washed in TBS-T and re-probed with antibodies. Relative expression levels of proteins were normalized to endogenous control GAPDH using ImageJ software 1.52v (National Institutes of Health).

Immunohistochemistry (IHC). Tissue sections $(5 \mu \mathrm{m})$ were stained with hematoxylin and eosin. Automatic IHC was performed with an automated immunostainer apparatus (BenchMark GX; Roche Diagnostics) using iVIEW DAB detection kit (Ventana) and primary antibodies specific for OGT (Sigma-Aldrich; Merck KGaA; cat. no. DM17; rabbit; 1:200), FASN (Abcam; cat. no. ab99359; rabbit; 1:100) and $O$-GlcNAc (Novus Biologicals RL2; mouse; 1:200). Antigen retrieval was performed using $\mathrm{CC} 1$ antigen retrieval buffer (Ventana Medical Systems) for $30 \mathrm{~min}$ at $95^{\circ} \mathrm{C}$. Specificity was checked by control staining performed in the absence of primary antibody. Images of whole tissue sections were obtained using an Axioscan Z1 microscope slide scanner (Zeiss AG). Immunostaining score was established by the expert pathologist Dr Rybarczyk. Staining intensity was analyzed using the percentages of stained hepatocytes (tumoral or not) multiplied by the intensity score as follows: 0 (no staining), 1+ (weak staining), 2+ (moderate staining) and 3+ (strong staining). We obtained a final score for each tissue ranging from 0 to 3 .

mRNA extraction and RT- $q P C R$ analysis. mRNA extraction was performed using the Nucleospin 'DNA, RNA and protein purification' kit (Macherey Nagel) according to the manufacturer's instructions. Quantification of RNA levels and reverse transcription were performed as previously described (7). The FASN, OGT and SREBP transcripts were analyzed by RT-qPCR using Mx4000 Multiplex Quantitative PCR system (Stratagene). Each PCR reaction contains $12.5 \mu \mathrm{l}$ of SyberGreen, $300 \mathrm{nM}$ of each primer and $2 \mu \mathrm{l}$ of cDNA for a total volume of $25 \mu \mathrm{l}$. The following program was followed: Segment 1 ( 1 cycle), $10 \mathrm{~min}$ at $95^{\circ} \mathrm{C}$; segment 2 (40 cycles), $30 \mathrm{sec}$ at $95^{\circ} \mathrm{C}, 30 \mathrm{sec}$ at $56^{\circ} \mathrm{C}$ for OGT and SREBP, and at $60^{\circ} \mathrm{C}$ for FASN, and $30 \mathrm{sec}$ at $72^{\circ} \mathrm{C}$; segment 3 (1 cycle), $1 \mathrm{~min}$ at $95^{\circ} \mathrm{C}, 30 \mathrm{sec}$ at $56^{\circ} \mathrm{C}$ for OGT and SREBP, and at $60^{\circ} \mathrm{C}$ for FASN, and $30 \mathrm{sec}$ at $95^{\circ} \mathrm{C}$. Data were normalized and expressed using the $2^{-\Delta \Delta \mathrm{CT}}$ method (13). The sequences of the primers are presented in Table II.

Statistical analysis. Data were presented are the means \pm standard error of the man of at least three independent experiments. Data were compared using one-way ANOVA and Student's t-test. Correlation analysis was done using Pearson correlation test (with the calculation of correlation coefficient $r$, coefficient of determination $\mathrm{R}^{2}$ and $\mathrm{P}$-value). Statistical analyses were performed using Excel 2019 (Microsoft Corporation) and Graph-Pad Prism 8.0 (GraphPad Software, Inc.) software.

\section{Results}

Exploration of transcriptome databases revealed that FASN and OGT gene expression are higher in cancers, including liver cancer. It is considered that FASN and $O G T$ are expressed in all human tissues but at various levels. To highlight the importance of FASN and OGT in physiological processes, we first checked gene expression levels of both enzymes in 54 healthy tissues (from 1,000 people) using GTExPORTAL (Fig. 1A). FASN and OGT were widely expressed over numerous tissues and organs. FASN content was higher in adipose tissue (due to visceral fat accumulation) and in mammary tissue, especially during lactation. Regarding OGT, the expression levels were more homogenous over tissues, although a stronger expression was observed in cerebellum, lung, spleen, thyroid, tibial nerve and female tissues and organs (cervix, fallopian tube, ovary, uterus and vagina; Fig. 1A). Since both enzymes are thought to be drivers of carcinogenesis, we next explored the GEPIA 2 web server to analyze their mRNA levels expressed as RNA-Seq by 

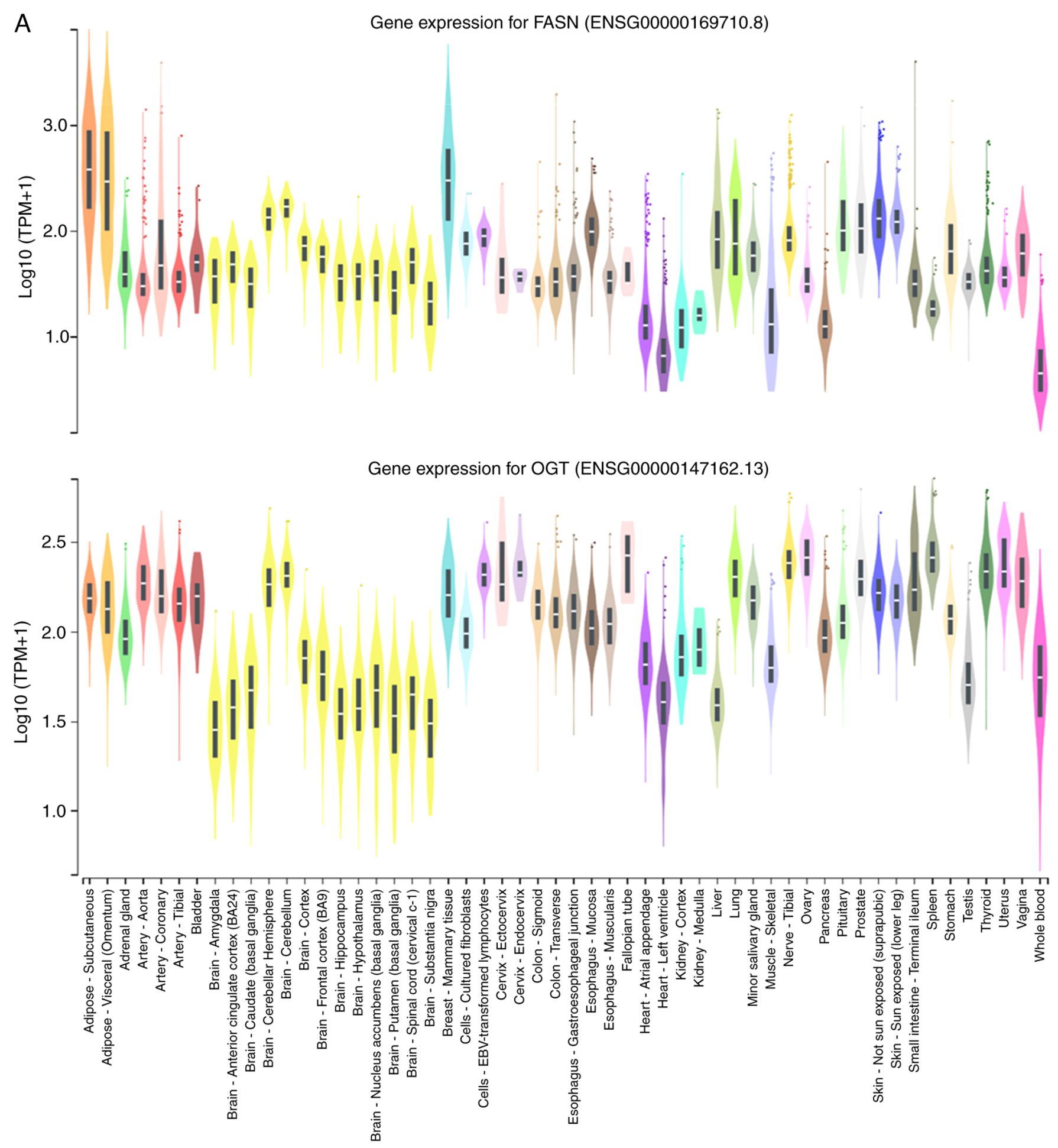

Figure 1. Continued.

Expectation-Maximization $(\log 2)$ in a wide variety of tumors (TCGA normal) compared with healthy tissues (TCGA normal and GTEx datasets; Fig. 1B). The results demonstrated that mRNA encoding FASN was increased in the following tumor tissues: BLCA, CESC, COAD, DLBC, LIHC, OV, PAAD, PRAD, READ, TGCT, THYM, UCEC and UCS. Conversely, it was significantly decreased in LAML and THCA (Fig. 1B). Significant increase in OGT expression was observed in CHOL, DLBC and LAML, and decrease in ACC, BRCA, CESC, COAD, LUAD, LUSC, OV, PAAD, PRAD, READ,
SKCM, THCA, THYM, UCEC and UCS. A non-significant decrease in OGT expression was also visible in LIHC, which was consistent with a previous study (7). Taken together, these findings demonstrated a significant decrease in OGT mRNA level in tumor tissues while FASN mRNA content tended to increase in tumor tissues.

FASN is highly expressed in cancer-derived cell lines compared with non-cancerous cell lines. We analyzed the expression of FASN and OGT in the three different cell lines derived from 

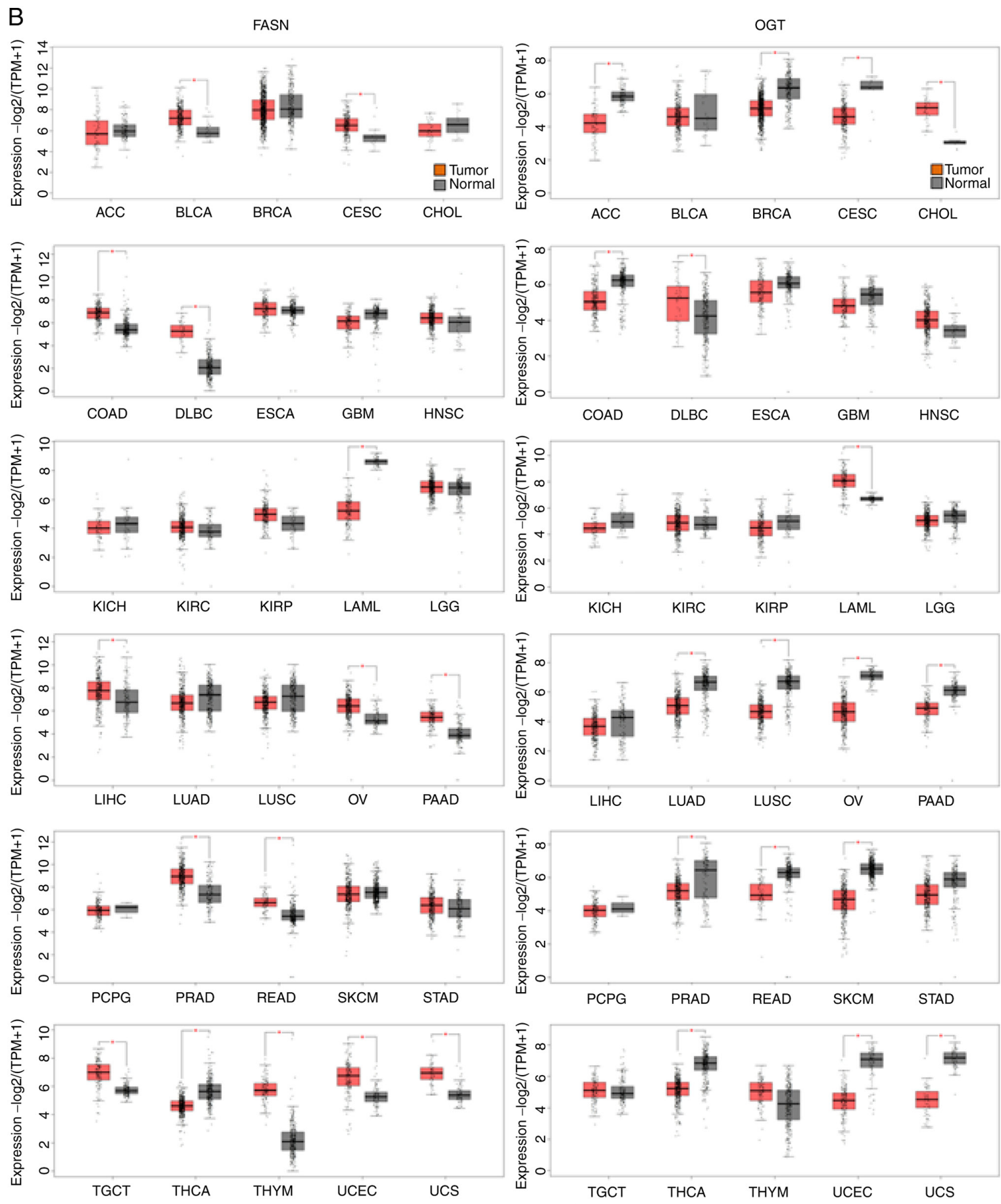

Figure 1. Evaluation of FASN and OGT gene expressions in (A) normal and (B) cancer human tissues using GTEx Portal and GEPIA2 respectively. Sample sizes (number of patients) were as follows in (A): AS, 663; AV, 541; AG, 258; AA, 432; AC, 240; AT, 663; B, 21; BA, 152; BACC, 176; BCBG, 246; BCH, 215; BCe, 241; BCo, 255; BFC, 209; BHi, 197; BHy, 202; BNABG, 246; BTBG, 205; BSCC, 159; BSN, 139; BMT, 459; CCF, 504; CEBVTL, 174; CEc, 9; CEn, 10; CS, 373; CT, 406; EGJ, 375; EMuc, 555; EMus, 515; FT, 9; HAA, 429; HLV, 432; KC, 85; KM, 4; Li, 226; Lu, 578; MSG, 162; MS, 803; NT, 619; O, 180; Pa, 328; Pi, 283; Pr, 245; SNSE, 604; SSE, 701; SITI, 187; Sp, 241; St, 359; Te, 361; Th, 653; U, 142; V, 156; WB, 755. ACC, adrenocortical carcinoma; BLCA, bladder urothelial carcinoma; BRCA, breast invasive carcinoma; CESC, cervical squamous cell carcinoma and endocervical adenocarcinoma; CHOL, cholangiocarcinoma; COAD, colon carcinoma; DLBC, lymphoid neoplasm diffuse large-B cell lymphoma; ESCA, esophageal carcinoma; GBM, glioblastoma multiforme; HNSC, head and neck squamous cell carcinoma; KICH, kidney chromophobe; KIRC, kidney renal clear cell carcinoma; KIRP, kidney renal papillary cell carcinoma; LAML, acute myeloid leukemia; LGG, brain lower grade glioma; LIHC, liver hepatocellular carcinoma; LUAD, lung adenocarcinoma; LUSC, lung squamous cell carcinoma; OV, ovarian serous cystadenocarcinoma; PAAD, pancreatic adenocarcinoma; PCPG, pheochromocytoma and paraganglioma; PRAD, prostate adenocarcinoma; READ, rectum adenocarcioma; SKCM, skin cutaneous melanoma; STAD, stomach adenocarcinoma; TGCT, testicular germ cell tumors; THCA, thyroid carcinoma; THYM, thymoma; UCEC, uterine corpus endometrial carcinoma; UCS, uterine carcinosarcoma. ${ }^{*} \mathrm{P}<0.05$.

liver HepG2, Hep3B and IHH by western blotting (Fig. 2). The expression of FASN was more elevated in liver cancer cells compared with IHH hepatocytes. While not significant for the
HepG2 cells, the P-value was equal to 0.06 when data were compared with the non-cancerous cell line. No differences were found for $O$-GlcNAcylation and OGT expression (Fig. 2). 
A

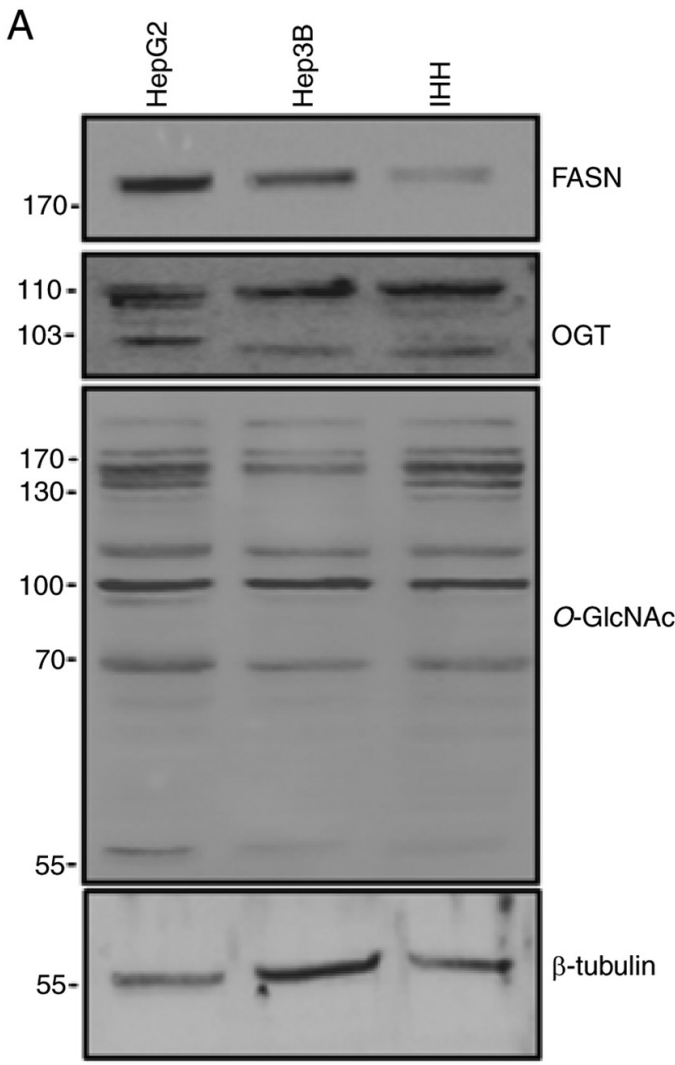

B

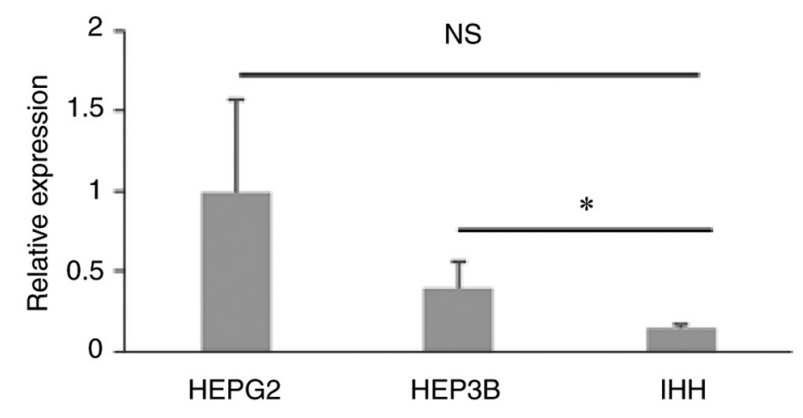

OGT/total proteins
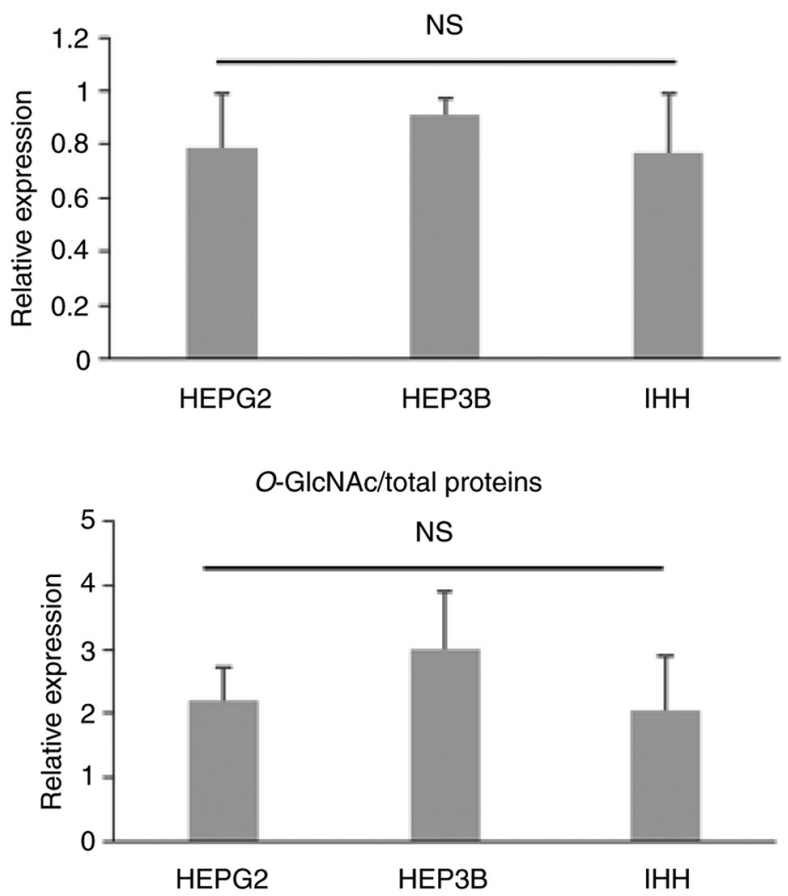

Figure 2. Analysis of FASN, OGT and $O$-GlcNAcylation contents in human hepatic cell lines. (A) Expression of FASN, OGT and $O$-GlcNAcylation was evaluated by western blot in three different hepatic cell lines, the liver cancer-derived cell lines HepG2 and Hep3B and the immortalized human hepatocytes IHH. (B) Quantification of three independent experiments from (A). " $\mathrm{P}<0.05$. NS, non-significant; FASN, fatty acid synthase; OGT, $O$-GlcNAc transferase; IHH, immortalized human hepatocyte.

FASN, OGT and O-GlcNAcylation expression is higher in human liver cancer tissues and FASN expression is correlated with activation of mTOR pathway. We analyzed the expression of FASN, OGT and $O$-GlcNAcylation in liver cancerous and non-cancerous tissues from 10 patients with moderately or well differentiated hepatocellular carcinoma (6 men and 4 women; Table I). We observed that FASN protein expression was more highly expressed in 6/10 tumor tissues when compared with non-tumor-adjacent tissues (Fig. 3A). Despite this increasing trend, there was no significant difference between tumor and non-tumoral tissues due to the high variability of FASN expression between patients (Fig. 3A and B). We previously reported that FASN expression is partly dependent upon the activation of the PI3K/AKT/mTOR pathway in hepatic cell lines and in livers from two different mice models, obese mice (ob/ob) and Phosphatase and tensin homolog-null mice (7). Like for FASN expression, we observed a higher activation of mTOR in liver tumors, although it was not significant due to the great inter-patient variability (Fig. 3A and B). Furthermore, FASN protein expression was positively correlated with mTOR activation in non-tumoral samples and corresponding cancer liver tissues as presented by the correlation analysis $(\mathrm{r}=0.8387$; $\mathrm{R}^{2}=0.7034$; P-value=0.0024; Fig. $3 \mathrm{C}$ ). However, the diversity of non-cancerous tissues damaged by different lesions (portal fibrosis, cirrhosis or normal phenotype) could explain at least partly the great inter-patient variability observed (Fig. 3A and B). An increase in OGT expression and a slight but significant increase in the $O$-GlcNAc expression were observed in human liver cancer tissues compared with non-cancerous tissues (Fig. 3A and B). The results from RT-qPCR demonstrated a slight increase in FASN and SREBP mRNA expression. Similar to the results from our previous study (7), OGT mRNA level tended to decrease in liver cancer tissues compared with non-cancerous tissues (Fig. 3D). The level of transcripts encoding FASN and OGT evolved in the same way as those found following exploration of GEPIA2 (Fig. 1B).

The expression of OGT, FASN and level of $O$-GlcNAcylation in human HCC tissues were evaluated 

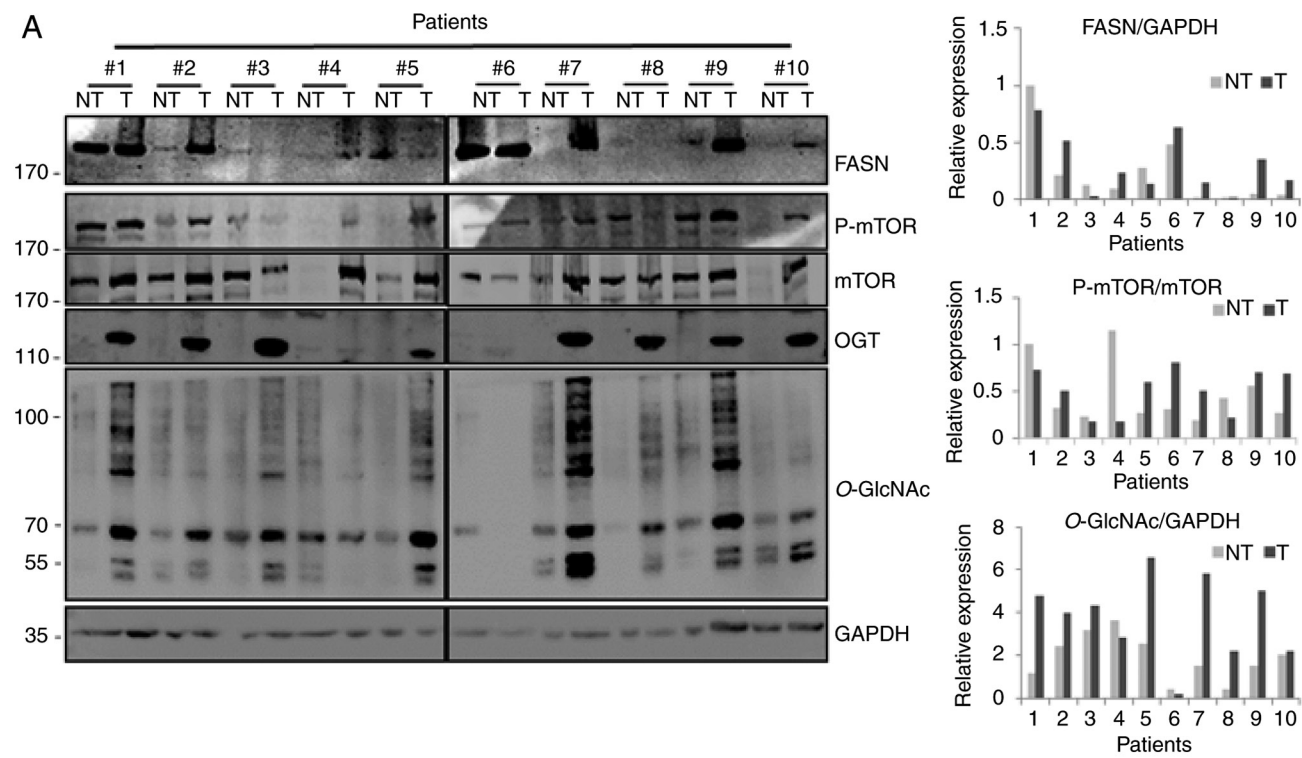

B
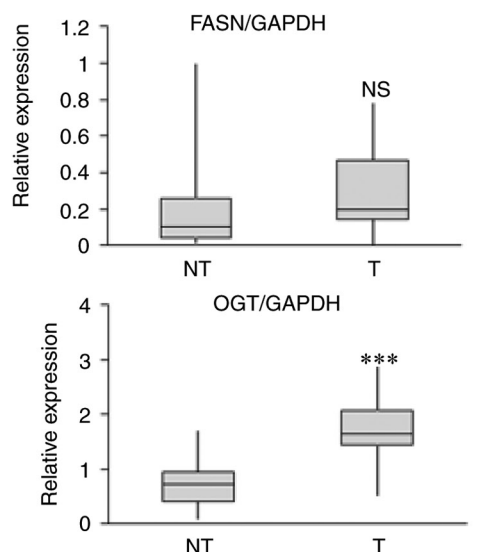

C 1
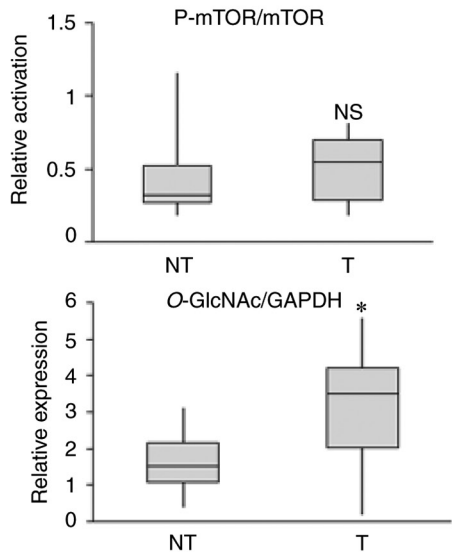

NT
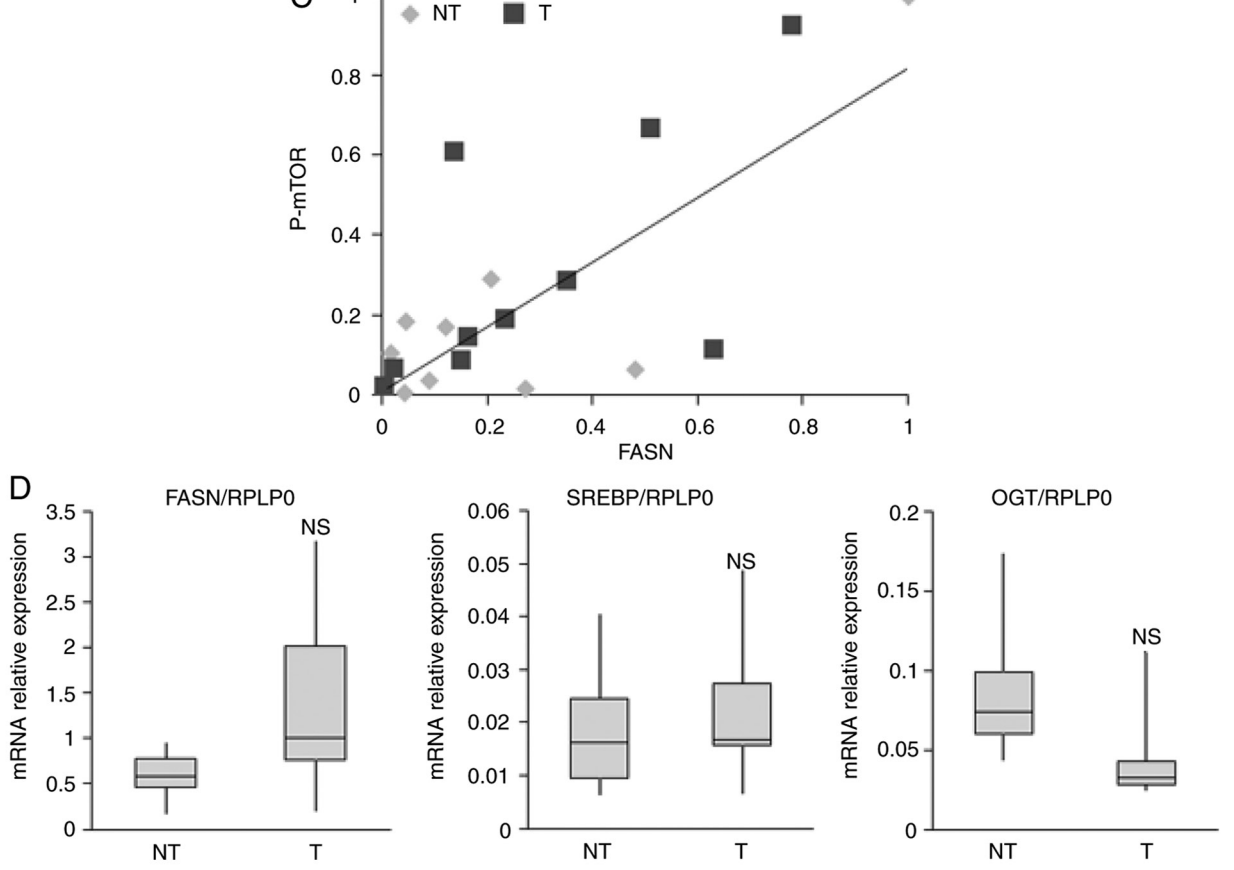

Figure 3. Analysis of FASN, OGT and $O$-GlcNAcylation expression and mTOR activation in human liver cancer tissues by western blot and RT-qPCR (A) Liver explants from 10 patients with hepatocellular carcinoma vs. non-tumoral adjacent tissues harboring various liver lesions were analyzed for FASN, OGT, $O$-GlcNAc, p-mTOR and mTOR expression by western blotting (left panel). Quantification of three independent experiments from (A) left panel (right panel). (B) Relative expression of FASN, OGT, $O$-GlcNAcylation and activation of mTOR pathway from 10 human liver tumor tissues and tumor-adjacent normal tissues. (C) Pearson correlation analysis between FASN expression and mTOR activation. (D) mRNA expression of OGT, FASN and SREBP measured by RT-qPCR. Values were normalized to RPLP0. ${ }^{*} \mathrm{P}<0.05$ and ${ }^{* * * *} \mathrm{P}<0.001$. NT, non-tumoral; T, tumoral; NS, non-significant; RT-qPCR, reverse transcription quantitative PCR; FASN, fatty acid synthase; OGT, $O$-GlcNAc transferase; mTOR, mechanistic/mammalian target of rapamycin; p, phosphorylated; SREBP, sterol responsive element binding protein; RLP0, ribosomal protein lateral stalk subunit P0. 
A
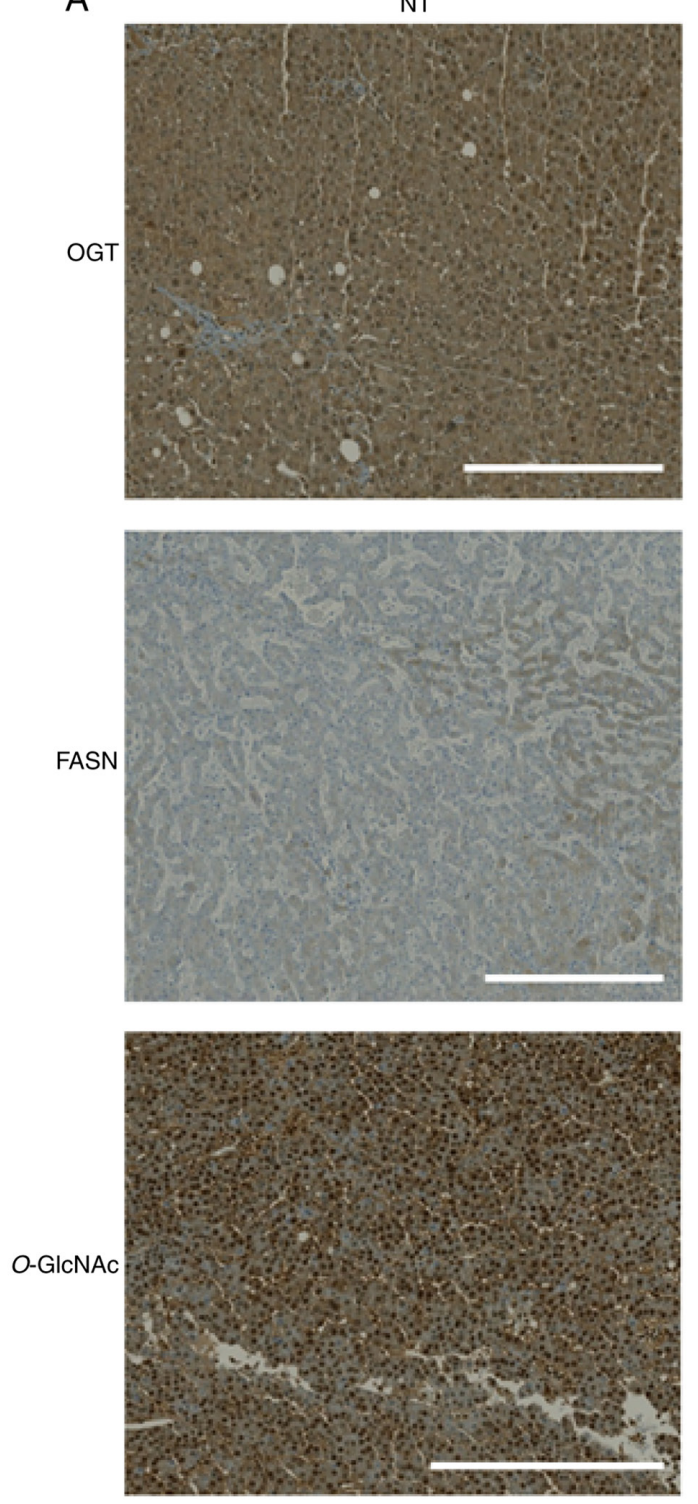

B

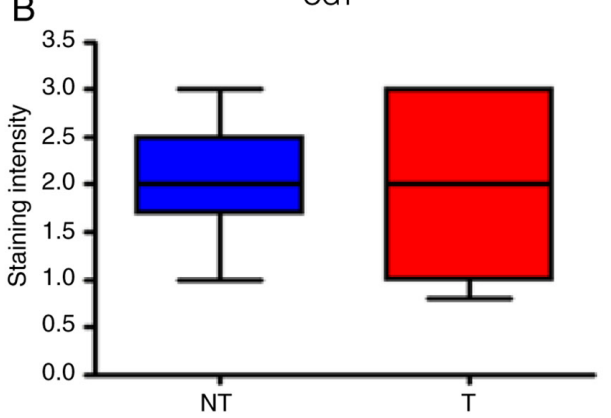

$\mathrm{T}$
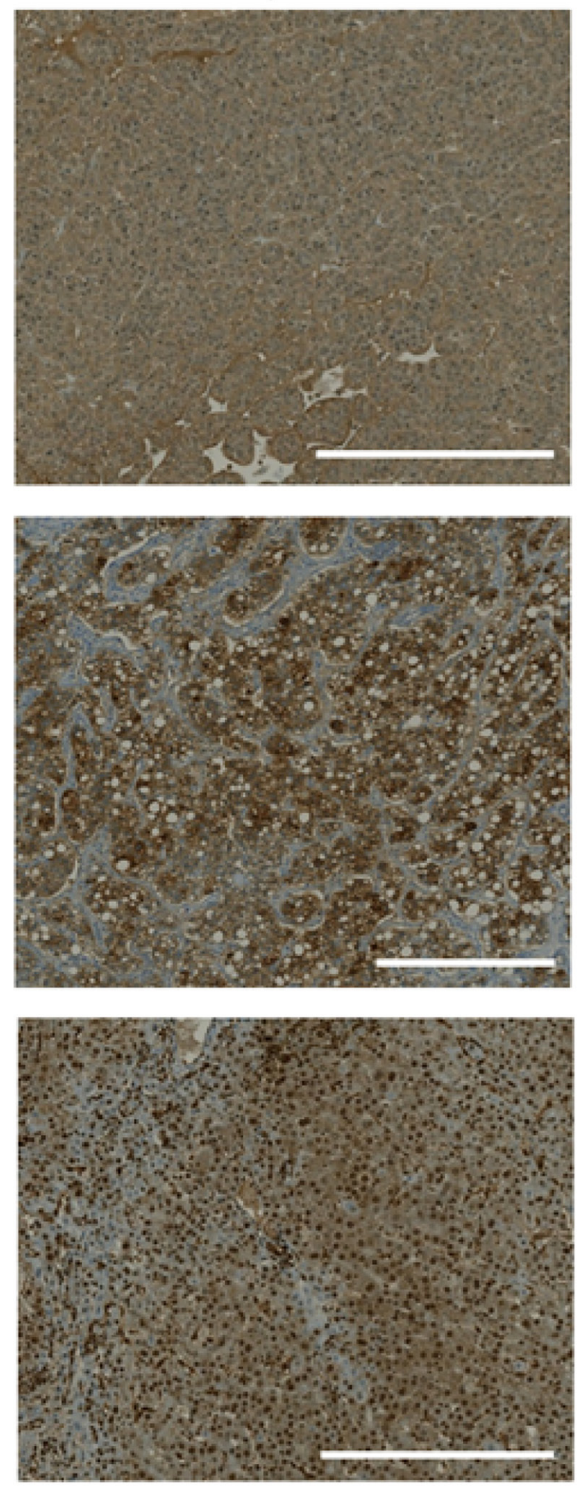

FASN

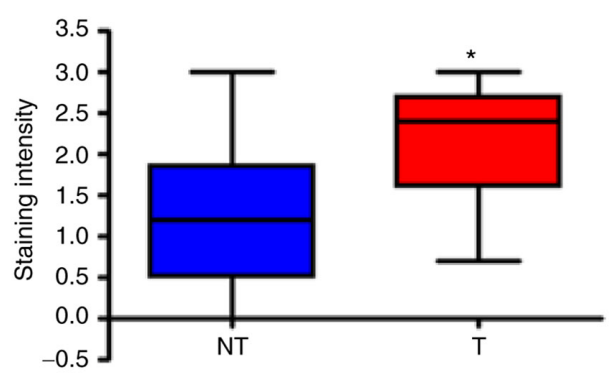

Figure 4. Analysis of FASN, OGT and $O$-GlcNAcylation expression in human liver cancer tissues by IHC. (A) Representative images of IHC staining in T and adjacent NT tissues. (B) IHC staining score was measured for OGT and FASN. Scale bar, $100 \mu \mathrm{m}$. "P<0.05. IHC, immunohistochemistry; NT, non-tumoral; T, tumoral; FASN, fatty acid synthase; OGT, $O$-GlcNAc transferase.

using IHC. In the 10 patients with HCC, a strongest FASN staining was observed in tumoral tissues compared with non-tumoral tissues $(2.2 \pm 0 / 21$ in tumor tissues vs. $1.24 \pm 0.26$ in normal tissues; $\mathrm{P}=0,02$; Fig. 4). FASN staining was mainly localized in the cytoplasm of tumoral cells with a little centrolobular increment of the intensity. No significative difference was observed for OGT expression between tumoral and non-tumoral tissues $(2.06 \pm 0.27$ in tumor tissues vs. $2.03 \pm 0.17$ in normal tissues; $\mathrm{P}=0,86)$. In both cases, the OGT strong staining was localized both in the nucleus and cytoplasm of the hepatocytes and was homogenous. Anti-O-GlcNAc staining was mainly nuclear. Similar strong intensity was observed in the tumor and normal tissues of the 10 patients with HCC (Fig. 4). 


\section{Discussion}

Cancer is one of the leading causes of morbidity and mortality worldwide. A growing body of evidence suggests that abnormalities in cell metabolism are closely related to the emergence and development of tumors. One of the aberrant metabolic pathways of tumor cells is the synthesis of fatty acids. FASN is the key enzyme involved in this process that provides energy for sustained proliferation of tumor cells (14). Therefore, an increased level of FASN has been observed in numerous cancers (3). FASN is also positively correlated with the aggressive stage of cancer and the poor prognosis. This increased lipogenesis provides cancer cells with some advantages in terms of proliferation, metastasis, survival and resistance to chemotherapy $(15,16)$.

In a previous study from our laboratory on hepatic lipogenesis, we reported that FASN is $O$-GlcNAcylated in a nutrition-dependent manner (6). The $O$-GlcNAcylation prevents the proteasomal degradation of FASN and increases therefore its expression and subsequent activity. In addition, we demonstrated in two independent studies that FASN expression is dependent on the catalytic activity of OGT and activation of mTOR in proliferating liver cancer cells, which is believed to promote hepatic carcinogenesis (7), and that mTOR and $O$-GlcNAcylation regulate each other (10) as previously described (9).

The present study demonstrated that FASN was more strongly expressed in the human HCC cell lines HepG2 and Hep3B compared with the immortalized human hepatocyte IHH cell line, which was not the case for OGT. This observation contrasts with what we previously observed in colon cell lines in which the glycosyltransferase is higher for cancer cell lines (17), and with Reginato's group for breast cancer cells (18). Thus, generalizing the elevation of OGT and $O$-GlcNAcylation in all cell types should not be done and a case-by-case study is essential. Furthermore, the use of a normal liver cell line such as THLE-3 would be helpful in a near future to push forward our investigations on this topic.

The present study also focused on the evaluation of FASN, OGT and $O$-GlcNAc expression and the activation of mTOR in 10 human HCC and non-tumoral adjacent tissues from 6 men and 4 women. By using western blotting, we demonstrated that, conversely to hepatic cell lines, the expression of OGT and $O$-GlcNAcylation was strongly elevated in liver cancer tissues compared with non-tumoral tissues, as previously demonstrated in colon tissues (19). It was previously reported by IHC that $O$-GlcNAcylation is significantly elevated in HCC tissues from patients treated with liver transplantation compared with health liver tissues (20), and that OGT and $O$-GlcNAcylation levels are higher in colon tumor tissues compared with tumor-adjacent normal tissues (19). In the liver cancer and adjacent non-tumoral tissues form the present study, no correlation between OGT mRNA and protein levels was reported; however, a decreasing trend was observed in OGT mRNA level. Regarding FASN protein expression, the results demonstrated that FASN was more highly expressed in 6 out of the 10 liver tumor tissues compared with non-tumor-adjacent tissues. Although the tendency to increase was the same, a high variability on FASN expression between patients was observed, which was probably due to the different types of liver lesion in the tissues (portal fibrosis or cirrhosis vs. normal phenotype). Thus, while non-significant, there was an increase in FASN expression between liver cancer tissues and non-tumoral tissues, these differences being highly heterogeneous from one patient to another. The mRNA encoding SREBP was also evaluated, which is the master transcription factor driving FASN expression. While transcripts level tended to increase, no significant changes was noticed, which was in accordance with our previous study (7). Overall, no sex differences regarding FASN, OGT and $O$-GlcNAc expression or activation of the mTOR pathway were observed in the present study. Furthermore, no difference was observed between the non-tumor tissues either, regardless of the lesion (portal fibrosis or cirrhosis vs. normal phenotype). However, a larger number of patients would help reinforcing these observations.

At the molecular level, we previously demonstrated that FASN depends on both catalytic activities of OGT and mTOR in liver proliferative cancer cells (7). Although the total level of mTOR can vary between patients, the present study demonstrated that FASN expression was correlated with the activation of mTOR pathway rather than with $O$-GlcNAcylation, conversely with what we formerly reported in cultured cells (7). These findings were in accordance with a previous study claiming that mTOR activation is highly variable in human liver tissues (21). In addition, we showed in a precedent paper that blocking FASN with the small-molecule inhibitor $\mathrm{C} 75$ can inhibit mTOR activation as well as OGT level and activity in HepG2 liver cancer cells, thus reducing cancer cell proliferation (7). These findings suggested that tumor-associated FASN, by conferring growth and survival advantages rather than functioning as an anabolic energy-storage pathway, may necessarily be associated with the history of human cancers.

By using IHC, increase in FASN expression in tumoral tissues compared with non-tumoral tissues was correlated with the non-significant increase of mRNA level in tumoral tissues. However, the lack of OGT significant difference in contrast with the western blotting results could have been attributed to a default of protein extraction during western blot or/and a resistance to antibodies penetrance in IHC. It would be of particular interest to confront these results to a staining of FASN and OGT in fibrotic or cirrhotic but non-cancerous liver samples, in order to focus only on the impact of these lesions on the expression of the two enzymes.

In summary, the present study demonstrated that increased FASN expression was associated with tumorigenesis, although the low number of tumor samples used was a limitation. The use of a larger cohort of patients will therefore be one of our priorities in future investigation. The expression of this key-metabolic enzyme was also correlated with mTOR pathway activation and more partially with OGT activity, both being known to be increased in human cancers. The results from the present study also highlighted that the analysis of identical samples by different experimental strategies could result in notable differences in interpretation, thus reinforcing the need to use different methods of analysis when studying tissues that are more complex than cell lines in culture.

\section{Acknowledgements}

The authors would like to thank Dr. Amélie Decourcelle (Université de Lille, CNRS, Inserm, CHU Lille, UMR9020- 
U1277-CANTHER-Cancer Heterogeneity, Plasticity and Resistance to Therapies, F-59000 Lille, France) for her help preparing liver lysates from patients. The authors would also like to thank Dr. Sylvie Janas, Dr. Laurence Wicquart and Professor Emmanuelle Leteurtre, scientific manager of the tumorotheque ALLIANCE-CANCER (Centre de Biologie-Pathologie, Lille) for providing the patient tissues.

\section{Funding}

This research was supported by the University of Lille, the 'Centre National de la Recherche Scientifique (CNRS)' and the Ligue Nationale Contre le Cancer (grant nos. 215348 and 215586 for comité du Nord). SR is a recipient of a fellowship from the 'Ministère de l'Enseignement Supérieur et de la Recherche' and from the 'Région Hauts-de-France'.

\section{Availability of data and materials}

All data generated or analyzed during this study are included in this published article.

\section{Authors' contributions}

NJ, IEYB and TL conceptualized the study. SR, NV and BD designed the methodology. SR, NV and BD performed the experiments. SR, PR, NJ and TL analyzed the data. SR and TL wrote the manuscript. All authors reviewed, read and approved the final manuscript. TL supervised the study. NJ, IEYB and TL confirm the authenticity of all the raw data.

\section{Ethics approval and consent to participate}

This study (approval no. CSTMT276) was approved by the relevant ethics committee (ALLIANCE-CANCER Tumorotheque-DC 2008-620) on December 2, 2020. Patients provided writing informed consent for the use of their samples in scientific research.

\section{Patient consent for publication}

Not applicable.

\section{Competing interests}

The authors declare that they have no competing interests.

\section{References}

1. Smith S, Witkowski A and Joshi AK: Structural and functional organization of the animal fatty acid synthase. Prog Lipid Res 42: 289-317, 2003.

2. Swinnen JV, Van Veldhoven PP, Timmermans L, De Schrijver E, Brusselmans K, Vanderhoydonc F, Van de Sande T, Heemers H, Heyns W and Verhoeven G: Fatty acid synthase drives the synthesis of phospholipids partitioning into detergent-resistant membrane microdomains. Biochem Biophys Res Commun 302: 898-903, 2003
3. Menendez JA and Lupu R: Fatty acid synthase and the lipogenic phenotype in cancer pathogenesis. Nat Rev Cancer 7: 763-777, 2007.

4. Hanover JA, Yu S, Lubas WB, Shin SH, Ragano-Caracciola M, Kochran J and Love D: Mitochondrial and nucleocytoplasmic isoforms of O-linked GlcNAc transferase encoded by a single mammalian gene. Arch Biochem Biophys 409: 287-297, 2003.

5. Fardini $Y$, Dehennaut $V$, Lefebvre $T$ and Issad $T$ : O-GlcNAcylation: A New cancer hallmark? Front Endocrinol (Lausanne) 4: 99, 2013.

6. Baldini SF, Wavelet C, Hainault I, Guinez C and Lefebvre T: The nutrient-dependent $\mathrm{O}-$ GlcNAc modification controls the expression of liver fatty acid synthase. J Mol Biol 428: 3295-3304, 2016.

7. Raab S, Gadault A, Very N, Decourcelle A, Baldini S, Schulz C, Mortuaire M, Lemaire Q, Hardivillé S, Dehennaut V, et al: Dual regulation of fatty acid synthase (FASN) expression by O-GlcNAc transferase (OGT) and mTOR pathway in proliferating liver cancer cells. Cell Mol Life Sci 78: 5397-5413, 2021.

8. Bar-Peled L and Sabatini DM: Regulation of mTORC1 by amino acids. Trends Cell Biol 24: 400-406, 2014.

9. Sodi VL, Khaku S, Krutilina R, Schwab LP, Vocadlo DJ, Seagroves TN and Reginato M: mTOR/MYC axis regulates O-GlcNAc transferase expression and O-GlcNAcylation in breast cancer. Mol Cancer Res 13: 923-933, 2015.

10. Very N, Steenackers A, Dubuquoy C, Vermuse J, Dubuquoy L, Lefebvre T and El Yazidi-Belkoura I: Cross regulation between mTOR signaling and O-GlcNAcylation. J Bioenerg Biomembr 50: 213-222, 2018.

11. Magaway C, Kim E and Jacinto E: Targeting mTOR and metabolism in cancer. Lessons and innovations. Cells 8: 1584, 2019.

12. Hua H, Kong Q, Zhang H, Wang J, Luo T and Jiang Y: Targeting mTOR for cancer therapy. J Hematol Oncol 12: 71, 2019.

13. Livak KJ and Schmittgen TD: Analysis of relative gene expression data using real-time quantitative PCR and the 2(-Delta Delta C(T)) method. Methods 25: 402-408, 2001.

14. Baldini SF and Lefebvre T: O-GlcNAcylation and the metabolic shift in high-proliferating cells: All the evidence suggests that sugars dictate the flux of lipid biogenesis in tumor processes. Front Oncol 6: 6, 2016.

15. Huang $C$ and Freter $C$ : Lipid metabolism, apoptosis and cancer therapy. Int J Mol Sci 16: 924-949, 2015.

16. Menendez $\mathbf{J}$ and Lupu R: Fatty acid synthase (FASN) as a therapeutic target in breast cancer. Expert Opin Ther Targets 21: 1001-1016, 2017.

17. Steenackers A, Olivier-Van Stichelen S, Baldini SF, Dehennaut V, Toillon RA, Le Bourhis X, El Yazidi-Belkoura I and Lefebvre T: Silencing the nucleocytoplasmic O-GlcNAc transferase reduces proliferation, adhesion, and migration of cancer and fetal human colon cell lines. Front Endocrinol (Lausanne) 7: 46, 2016.

18. Caldwell SA, Jackson SR, Shahriari KS, Lynch TP, Sethi G, Walker S, Vosseller K and Reginato MJ: Nutrient sensor $\mathrm{O}$-GlcNAc transferase regulates breast cancer tumorigenesis through targeting of the oncogenic transcription factor FoxM1. Oncogene 29: 2831-2842, 2010.

19. Olivier-Van Stichelen S, Dehennaut V, Buzy A, Zachayus JL, Guinez C, Mir AM, El Yazidi-Belkoura I, Copin MC, Boureme D, Loyaux D, et al: O-GlcNAcylation stabilizes $\beta$-catenin through direct competition with phosphorylation at threonine 41 . FASEB 28: 3325-3338, 2014.

20. Zhu Q, Zhou L, Yang Z, Lai M, Xie H, Wu L, Xing C, Zhang F and Zheng S: O-GlcNAcylation plays a role in tumor recurrence of hepatocellular carcinoma following liver transplantation. Med Oncol 29: 985-993, 2012.

21. Meng Z, Li T, Ma X, Wang X, Van Ness C, Gan Y, Zhou H, Tang J, Lou G, Wang Y, et al: Berbamine inhibits the growth of liver cancer cells and cancer-initiating cells by targeting $\mathrm{Ca}^{2+} /$ calmodulin-dependent protein kinase II. Mol Cancer Ther 12: 2067-2077, 2013.

This work is licensed under a Creative Commons Attribution-NonCommercial-NoDerivatives 4.0 International (CC BY-NC-ND 4.0) License. 